\title{
Influence of Plasmon Excitations in Au Nanoparticles upon Fluorescence and Photostability of Photosynthetic Complexes
}

\author{
Bartosz Krajnik $^{1}$, Nikodem Czechowski ${ }^{1}$, D. Piatkowski ${ }^{1}$, Sebastian Mackowski ${ }^{*}$, \\ Eckhard Hofmann ${ }^{2}$, Stefan Pichler ${ }^{3}$, Wolfgang Heiss ${ }^{3}$ \\ ${ }^{1}$ Institute of Physics, Faculty of Physics, Astronomy and Informatics, Nicolaus Copernicus University, Torun, Poland \\ ${ }^{2}$ Department of Biology and Biotechnology, Ruhr-University Bochum, Bochum, Germany \\ ${ }^{3}$ Institut für Halbleiter-und Festkörperphysik, Universität Linz, Linz, Austria \\ Email: ${ }^{*}$ mackowski@fizyka.umk.pl
}

Received November 19, 2012; revised December 20, 2012; accepted December 28, 2012

\begin{abstract}
Fluorescence spectroscopy is applied to study the influence of plasmon excitations in spherical Au nanoparticles on the optical properties of chlorophyll-containing light-harvesting complexes. The separation between the two nanostructures is controlled via silica layer with varied thickness. We observe strong increase of the emission intensity for a 12$\mathrm{nm}$-thick spacer and the increase is accompanied with shortening of the fluorescence lifetime, which allows us to separate contributions of absorption and emission rate enhancement. At the same time we find an increase of photobleaching. These findings are interpreted as a result of spectral overlap between plasmon resonance and chlorophyll fluorescence.
\end{abstract}

Keywords: Plasmonics; Fluorescence; Photosynthesis

\section{Introduction}

Metal-enhanced fluorescence [1] has been a subject of very intense research in recent years with several important ideas demonstrated that hold merit not only for understanding fundamental processes at the interface between metallic nanostructures and fluorophores but also for applications in medical imaging [2], high-resolution optical spectroscopy [3], biosensors [4], and photovoltaics [5]. The effect of fluorescence enhancement for emitters placed in the vicinity of metallic nanoparticles is attributed to plasmonic excitations, i.e. collective oscillations of free electrons upon optical excitation. Depending on a size and shape of the nanoparticles, the plasmon resonance can cover pretty large spectral region: from $400 \mathrm{~nm}$ for silver nanoparticles and silver island films, through $550 \mathrm{~nm}$ characteristic for gold nanoparticles, up to near infrared region, where plasmon resonances of nanorods, nanotriangles and nanoshells can be shifted through precise control of the morphology [6,7]. Among methods applied for fabrication of metallic nanoparticles with defined geometry wet chemistry and electron beam lithography have been particularly efficient and versatile.

In addition to spectral properties of metallic nanoparticles, and of fluorophores as well, the interaction of dipole moments of fluorophores with plasmonic excitations exhibits strong dependence upon the distance $[8,9]$. There are essentially three regimes: in the first case, where the separation is larger than $50 \mathrm{~nm}$ the interaction is too weak to induce any changes in the optical properties of fluorophores. As the separation gets reduced, reaching the range of approximately $10-30 \mathrm{~nm}$, plasmonic excitations in metallic nanoparticles induce increase of absorption and fluorescence rates. These effects contribute to enhancement of the fluorescence intensity measured in experiment. For the smallest distances, below $5 \mathrm{~nm}$, a non-radiative energy transfer from fluorophores to metallic nanoparticles dominates the dynamics in the system and overcomes further increase of fluorescence and absorption rates. As a result, the fluorescence intensity gets dramatically reduced. The increased role of the non-radiative recombination manifests itself by strong shortening of the fluorescence decay time measured for fluorophores placed extremely close to metallic nanoparticles. The energy is later transformed into heat. The distance dependence of the fluorescence intensity in hybrid nanostructures composed of metallic nanoparticles has been demonstrated on a single molecule level as well as on highly concentrated samples.

Control of the separation between metallic nanoparticles and fluorophores has been achieved either via covalent binding using polymers [4] or biotin-streptavidin conjugation $[10,11]$. These approaches are suitable pri- 
marily for solutions of nanostructures. Another strategy is based on covering metallic nanoparticles with dielectric shells, for instance $\mathrm{SiO}_{2}$ thin layers. It is however quite difficult to control the thickness of such layers to the precision required for applications in plasmonics [12]. In the case of planar hybrid nanostructures metallic nanoparticles have been covered with dielectric layers using evaporation techniques. Advancement in technology of nanostructures has enabled to control the thickness of dielectric layers fabricated using these methods with great accuracy. In particular, it has been recently shown that spherical metallic nanoparticles covered with silica layers with defined thickness can feature all regimes of plasmonic interactions: from quenching to strong enhancement [9].

Interaction between plasmon excitations in metallic nanoparticles and fluorophores leads not only to enhancement of the fluorescence intensity, but it can also modify photostability of the chromophores. In several experiments, interactions with plasmons were shown to improve the photostability, as demonstrated even at a single nanoparticle level [13], but there are also many examples, where the opposite was observed [14].

In this work we fabricate a hybrid nanostructure composed of a monolayer of spherical gold nanoparticles and a light-harvesting complex from algae, peridinin-chlorophyll-protein (PCP). In order to control the separation between both constituents, the $\mathrm{Au}$ nanoparticles were covered with a silica layer with a thickness from 4 to 40 $\mathrm{nm}$. In this way we can probe the whole range of plasmonic interactions. The interaction between the pigments embedded in the complex with plasmonic excitations in the $\mathrm{Au}$ nanoparticles was probed using spectrally and temporally resolved fluorescence microscopy. The results indicate that by changing the spacer thickness we can demonstrate pronounced distance dependence of the fluorescence intensity. The maximum enhancement of a factor of 7 is obtained for the structure, in which the PCP complexes are separated from the metallic nanoparticles by $12 \mathrm{~nm}$. For thicker layers the strength of the interaction becomes weaker, while for the thinner spacer of 4 $\mathrm{nm}$ the intensity decreases presumably due to non-radiative energy transfer. The results are important for understanding the influence of various factors that determine the effect of plasmonic excitations upon the optical properties of biomolecules in the context of constructing hybrid nanostructures with biological functionality.

\section{Materials and Methods}

The PCP complexes were obtained according to protocol by Miller et al. [15]. Briefly, PCP apoprotein in $50 \mathrm{mM}$ Tris- $\mathrm{HCl} \mathrm{pH} 8.0$ solution was added to $25 \mathrm{mM}$ Tricine and $10 \mathrm{mM} \mathrm{KCl}(\mathrm{pH} \mathrm{7.6)}$, mixed with a stoichiometric amount of PCP pigments (peridinin and chlorophyll a) dissolved in ethanol. The sample was held in $4^{\circ} \mathrm{C}$ for 72 h. Reconstituted samples were equilibrated to $5 \mathrm{mM}$ Tricine with $2 \mathrm{mM} \mathrm{KCl} \mathrm{(pH} \mathrm{7.6)} \mathrm{by} \mathrm{passage} \mathrm{through} \mathrm{a}$ PD-10 column and bound to a column of DEAE Trisacryl. Reconstituted PCP was then removed with $5 \mathrm{mM}$ Tricine with $2 \mathrm{mM} \mathrm{KCl}$ (pH 7.6) containing $0.06 \mathrm{M} \mathrm{NaCl}$. Gold nanoparticles were synthesized using a reduction reaction and dispersed in toluene. The average diameter of the nanocrystals was $5 \mathrm{~nm}$.

Plasmonic substrates for fluorescence measurements were prepared by spin-coating solutions of the Au nanoparticles on clean glass coverslips. This procedure, as shown previously, yields a uniform monolayer of Au nanoparticles [16]. Next, the substrates were transferred to an ebeam evaporation system, where $\mathrm{SiO}_{2}$ layers with thicknesses from 4, to $40 \mathrm{~nm}$ were deposited. In order to form well-defined layers of PCP, the light-harvesting complexes were dispersed in polyvinylalcohol (PVA) polymer (Sigma Aldrich) and spin-coated on the silica surface. We applied $10 \mu \mathrm{L}$ of the PCP solution with a concentration of $0.2 \mathrm{mg} / \mathrm{mL}$ at speed of $2400 \mathrm{rpm}$ for 60 seconds, this procedure resulted in a thin, homogeneous layer of the PCP complexes. Experiments were carried out immediately after sample preparation.

Fluorescence measurements were performed with our home-build confocal microscope based on the Olympus infinity-corrected microscope objective LMPlan 50×, characterized with a numerical aperture of 0.5 [17]. Sample was placed on a XYZ piezoelectic stage (Physik Instrumente), which enables scanning of the sample surface to measure fluorescence intensity maps. Once a fluorescence map is collected, a point of interest can be identified on it, and both fluorescence spectrum and lifetime can be measured. The spectrum, dispersed using am Amici prism is measured with a CCD detector (Andor iDus DV 420A-BV). The spectral resolution of the setup is better than $1 \mathrm{~nm}$. Fluorescence lifetimes are measured using time-correlated single photon counting module (Becker \& Hickl) equipped with a fast avalanche photodiode (idQuantique id100-50) triggered by a laser pulse. The emission of the PCP complexes was excited with a laser at wavelength of $485 \mathrm{~nm}$, the exctiation power and repetition rate of $50 \mu \mathrm{W}$ with $20 \mathrm{MHz}$ were used respecttively. The fluorescence spectra were acquired with integrating time of $0.5 \mathrm{~s}$, fluorescence transients were collected for 60 seconds. For the latter, in order to isolate the emission of the PCP complexes we used a HQ670/10 (Chroma) bandpass filter.

\section{Results and Discussion}

The structure of the PCP light-harvesting complex was determined using X-ray crystallography [18] and is shown 


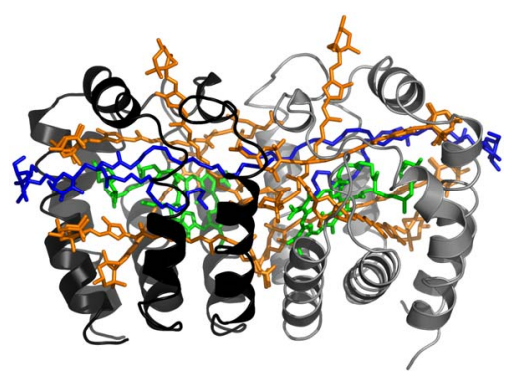

(a)

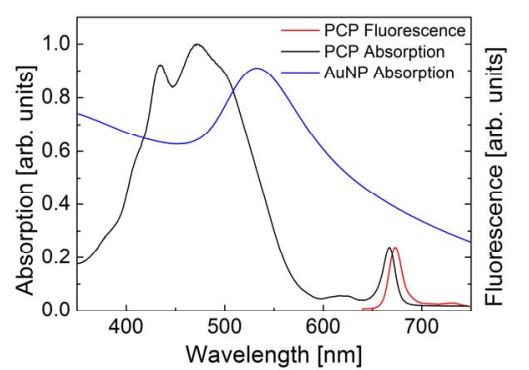

(b)

Figure 1. (a) Structure of the monomer of the PCP complex. Chlorophylls are colored green, peridnins are shown in orange, the lipid molecule is shown in blue. The pigments are embedded in a protein. PDB entry 3IIS; (b) Comparison of absorption spectrum of the PCP complex with the absorption spectrum of Au nanoparticles. The emission of the PCP is also shown.

each containing one chlorophyll $a(\mathrm{Chl} a)$, four peridinins (Per), and one digalactosyl diacyl glycerol (DGDG) lipid molecule. The distance between the Chls is $17.4 \AA$, while the whole PCP monomer diameter is about $4 \mathrm{~nm}$ with molecular weight about $32 \mathrm{kDa}$. The optical spectra of the PCP complexes used in the experiment are shown in Figure 1. Absorption spectrum is dominated by a broad band from $400 \mathrm{~nm}$ to $550 \mathrm{~nm}$. It is mainly associated with Per, which is a major absorbing pigment of the PCP complexes. Chl $a$ molecules absorb around $441 \mathrm{~nm}$ (Soret band) and $670 \mathrm{~nm}\left(\mathrm{Q}_{\mathrm{y}}\right.$ band). The broad and weak absorption around $620 \mathrm{~nm}$ originates from $\mathrm{Q}_{\mathrm{x}}$ absorption of the $\mathrm{Chl} a$ molecules. The absorption spectrum measured for Au nanoparticles is also shown in Figure 1, it features strong plasmon resonance at $540 \mathrm{~nm}$, but the resonance is pretty broad, extending considerably towards the emission of the PCP complex. The fluorescence of the PCP is associated with $\mathrm{Q}_{\mathrm{y}}$ band of the weakly coupled $[19,20] \mathrm{Chl} a$ pair and appears at $673 \mathrm{~nm}$. Due to solubility in water, high fluorescence quantum yield, and strong absorption in the spectral range similar to that of plasmon resonances in Au and Ag nanoparticles, the PCP complexes are very attractive biomolecules for studying interactions with metallic nanoparticles.

In Figure 2 we show schematics of the sample used for the study. The layer of spherical gold nanoparticles is covered with a silica spacer of a defined thickness $(4 \mathrm{~nm}$, $12 \mathrm{~nm}$, and $40 \mathrm{~nm}$ ). On top of the spacer a layer of the PCP complexes in a PVA matrix were deposited. The orientation of the PCP complexes on the surface ws random.

The evaluation of the plasmon induced modifications of the fluorescence properties of the PCP complexes was carried out in th following way. In the first step an intensity map was measured, the size of the map was about $100 \times 100 \mu \mathrm{m}$. For all spacer thicknesses the maps were quite uniform, with the most variation of the fluorescence intensity visible in the case of the 12-nm-thick silica spacer and the 4-nm-thick silica spacer. In the next step a series of fluorescence spectra and fluorescence transients

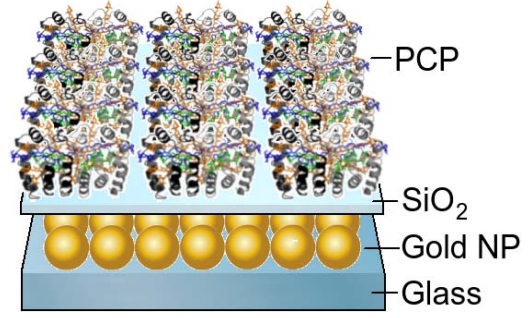

Figure 2. Schematic drawing of the hybrid nanostructure comprising gold nanoparticles and light-harvesting complexes. The thickness of the silica spacer was changed from 4 to $40 \mathrm{~nm}$.

in Figure 1. In its native form the PCP forms a trimer with each of the monomers composed of two N-domains, were collected. For each spacer we measured 50 spectra and 15 transients. In this way statistically significant information about fluorescence intensity as well as fluorescence decay time is obtained. Each measurement was carried out on a new spot on the sample that has not been illuminated previously in order to reduce any possible effects of the photobleaching.

In Figure 3 we show examples of fluorescence spectra measured for the samples with three different spacers. Each panel contains five spectra selected randomly from the sets that were measured. The first observation is that the overall shapes of the spectra are identical regardless of the thickness of the spacer. They are also identical to the fluorescence of the PCP complexes measured previously and published before [19]. The absence of any effect of the metallic nanoparticles that are in the vicinity of the light-harvesting complexes upon the shape of the emission spectrum strongly suggests that the protein remains intact and is completely functional, for instance, all the energy transfer pathways or interactions between the pigments are essentially unchanged. Importantly, we find strong dependence of the intensity of the PCP emission on the thickness of the silica spacer. In the case of the thickest spacer of $40 \mathrm{~nm}$, the intensity reaches 4000 cps, while for a 12-nm-thick spacer the intensities at maximum wavelength are above $25,000 \mathrm{cps}$. Further re- 


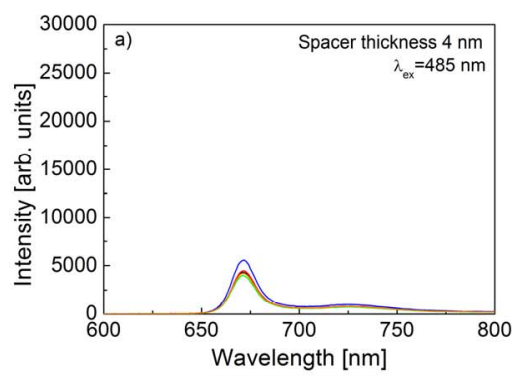

(a)

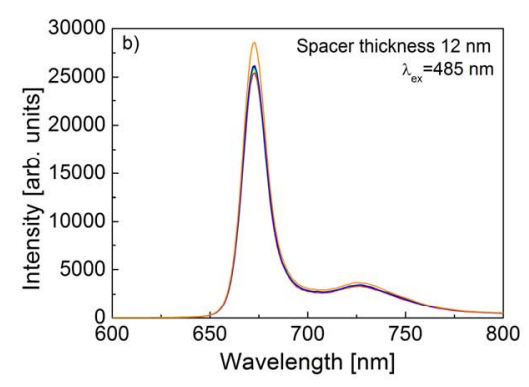

(b)

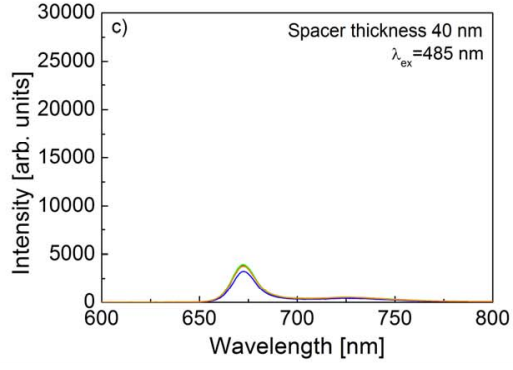

(c)

Figure 3. Representative fluorescence spectra measured for the PCP complexes deposited on Au nanoparticles covered with a silica spacer of (a) $4 \mathrm{~nm}$; (b) $12 \mathrm{~nm}$; and (c) $40 \mathrm{~nm}$. The spectra for any given spacer thickness of the $\mathrm{SiO}_{2}$ layer were taken out of several locations across the sample.

duction of the spacer thickness down to $4 \mathrm{~nm}$ results in decrease of the fluorescence intensity with a maximum values at about $5000 \mathrm{cps}$.

Fluorescence decay curves measured for the PCP complexes for these three structures provide a way to distinguish between the impact of various processes associated with plasmonic excitations upon the optical properties of the light-harvesting complexes. In Figure $\mathbf{4}$ we display a set of normalized fluorescence transients measured for the three different spacers. For each sample 10 curves are shown. The first observation is that for all three samples the ten decays collected are essentially identical: the data is essentially stacked on top of each other. There are however differences between hybrid nanostructures with varied spacer thickness. For the structure, where the thickness of the silica spacer was equal to $40 \mathrm{~nm}$ the decay is the longest, comparable to the results measured previously for isolated PCP complexes. The fastest decay is present for the structure with the spacer thickness of 4 $\mathrm{nm}$. The transient behavior measured for the PCP complexes separated from the Au nanoparticles by $12 \mathrm{~nm}$ of a silica layer, the decay is intermediate. The spacer dependence of the fluorescence decay measured for the PCP complexes deposited on a layer of Au nanoparticles is qualitatively different as compared with another studied case of the light harvesting complex LH2 from purple bacteria [9], where the lifetime has been found insensitive to the thickness of the spacer layer. In the case of the LH2 complex, however, the emission appears at $870 \mathrm{~nm}$, which is approximately $200 \mathrm{~nm}$ further to the red as compared to the PCP complex. Therefore, there is little spectral overlap between the plasmon resonance of the Au nanoparticles and the emission of the LH2 complex. As a consequence, there is essentially no coupling expected between the plasmon resonance in such Au nanoparticles and electronic transitions of the LH2 complex. In contrast, in the case of the PCP complexes, where the spectral overlap between the emission and plasmon resonance is much stronger, the excitation of plasmons in metallic nanoparticles induces modifications of radiative properties of the Chl $a$ embedded in the protein.

Quantitative analysis of the fluorescence spectra and transients in displayed in Figure 5 where histograms of the two parameters are plotted. Fluorescence lifetimes were calculated through fitting a single exponential decay to the experimental curve with the Levenberg-Marquad algorithm, while fluorescence intensities were obtained directly from the emission maximum at $673 \mathrm{~nm}$. The intensity histogram obtained for the 40-nm-thick silica spacer is very narrow with an average value of $3900 \mathrm{cps}$. The corresponding distribution of the fluorescence decay times reveals also quite uniform character, although the determination of the fluorescence decay time is less accurate $(\sim 200 \mathrm{ps})$. The average value of the decay time measured for the PCP complexes deposited $40 \mathrm{~nm}$ away from the layer of $\mathrm{Au}$ nanoparticles is $4.5 \mathrm{~ns}$. The value is somewhat longer than in the case of the PCP in solution (3.7 ns [19]), which could be due to the influence of the PVA matrix. Nevertheless, as all the samples studied in this work were prepared in exactly the same way, the comparison between the structures with varied spacer thickness is valid. Upon decrease of the spacer thickness to $12 \mathrm{~nm}$, the fluorescence intensity exhibits strong increase due to interaction with plasmon excitations in metallic nanoparticles, reaching an average value of 26,700 cps, which amounts to the enhancement factor of 6.8 . The increase of the fluorescence intensity is accompanied with a shortening of the fluorescence decay time. In the case of the 12-nm-thick structure, the average value of the fluorescence decay time of $3.1 \mathrm{~ns}$ was obtained, which is by approximately $30 \%$ shorter than for the reference structure with a 40-nm-thick spacer. Attributing the reduction of the decay time to increase of the radiative rate of the Chl $a$ molecules in the PCP complex, we can conclude that factor of 2 increase of the fluorescence intensity is due to radiative rate enhancement, while the rest is due to increase of absorption of the light-harvesting complex. We note that the distributions of the fluorescence intensity and lifetime obtained for the structure, where the PCP complexes were separated by $12 \mathrm{~nm}$ from 


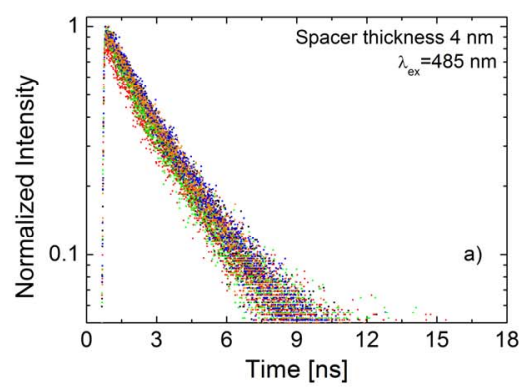

(a)

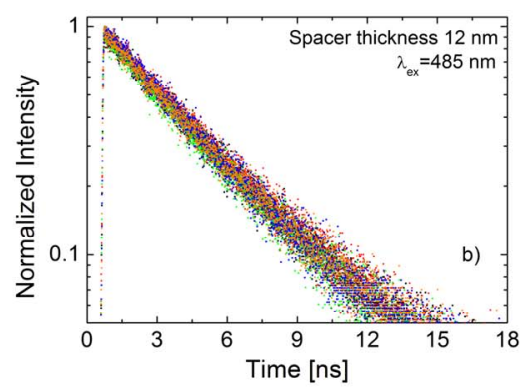

(b)

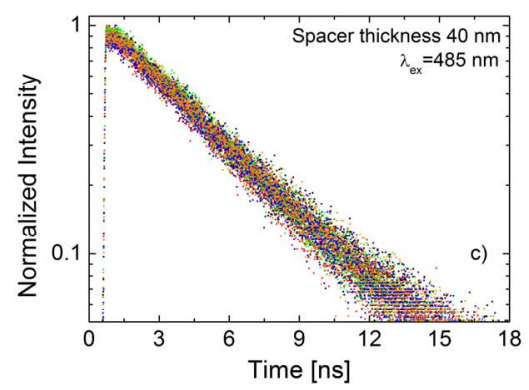

(c)

Figure 4. Representative normalized fluorescence transients measured for the PCP complexes deposited on Au nanoparticles covered with a silica spacer of (a) $4 \mathrm{~nm}$; (b) $12 \mathrm{~nm}$; and (c) $40 \mathrm{~nm}$. The transients for any given spacer thickness of the $\mathrm{SiO}_{2}$ layer were taken out of several locations across the sample.

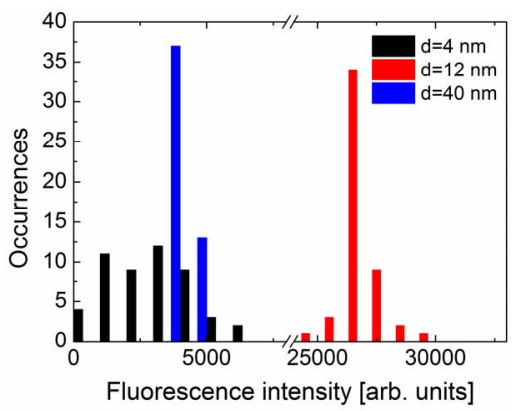

(a)

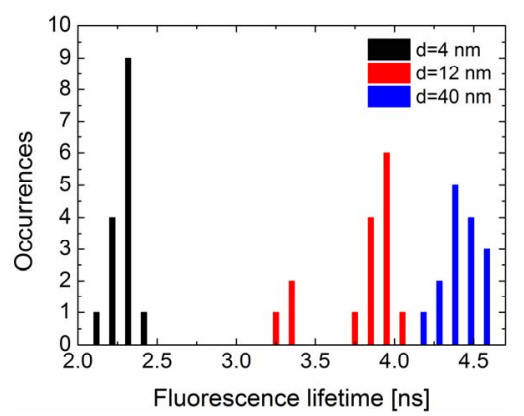

(b)

Figure 5. Histograms of the (a) fluorescence intensity and (b) fluorescence lifetimes measured for hybrid nanostructures with spacer thickness of $4 \mathrm{~nm}$ (black), $12 \mathrm{~nm}$ (red) and $40 \mathrm{~nm}$ (blue).

the layer of the Au nanoparticles are broader as compared to the reference structure with the 40-nm-thick silica spacer. The increased inhomogeneity of the sample is directly associated with a strong dependence of the plasmonic interactions upon the geometry of the structure, in particular the separation between the two constituents of the hybrid nanostructure. Even tiny changes in the thickness of the silica spacer may result in measurable changes of both the lifetime and intensity. This effect becomes even more pronounced for the structure with the thinest spacer of $4 \mathrm{~nm}$. In this case the average values of $2.3 \mathrm{~ns}$ and $3000 \mathrm{cps}$ for the lifetime and intensity were obtained, respectively and in particular the intensity diagram features strong broadening. The reduction of the fluorescence intensity coupled to the decrease of the fluorescence lifetime can be a result of non-radiative energy transfer from $\mathrm{Chl}$ molecules to the Au nanoparticles.

Spectroscopy of the hybrid nanostructure composed of spherical $\mathrm{Au}$ nanoparticles coupled to the PCP lightharvesting complexes reveals all important modes of interaction between fluorophores and plasmon excitations: from very little influence $(40 \mathrm{~nm})$ through strong increase of the fluorescence intensity $(12 \mathrm{~nm})$ up to fluorescence quenching $(4 \mathrm{~nm})$. We conclude that through precise engineering of the design, it is possible to achieve the desired functionality of such a structure, which is important for devising and testing alternative architecttures that involve plasmonically active nanostructures and biomolecules.

The final aspect of the experiment that we discuss is the role of photobleaching and the effect of the plasmonic excitations in metallic nanoparticles on it. The intensity of the fluorescence, in addition to being estimated directly from the spectra, can also be obtained by integrating the transient curve measured in a time-resolved experiment. The only difference between these two approaches is an integration time, which is $0.5 \mathrm{sec}$ and $60 \mathrm{sec}$, respectively. In the case of emitters that are not susceptible to photobleaching the results should be comparable, as indeed observed for the LH2 complexes coupled to $\mathrm{Au}$ nanoparticles in analogous structures [9]. In Figure 6 we show the result of integration of the fluorescence transients for structures with different silica spacers. While the intensities obtained for the sample with 4-nm-thick spacer are still the lowest (average value of 110), we observe almost no difference between the data obtained for the 40-nm-thick and 12-nm-thick samples. The average values of the emission intensity were 200 and 260, respectively, although the data for the 12$\mathrm{nm}$-thick sample exhibits still substantially broader distribution. The similarity of the results obtained for the 


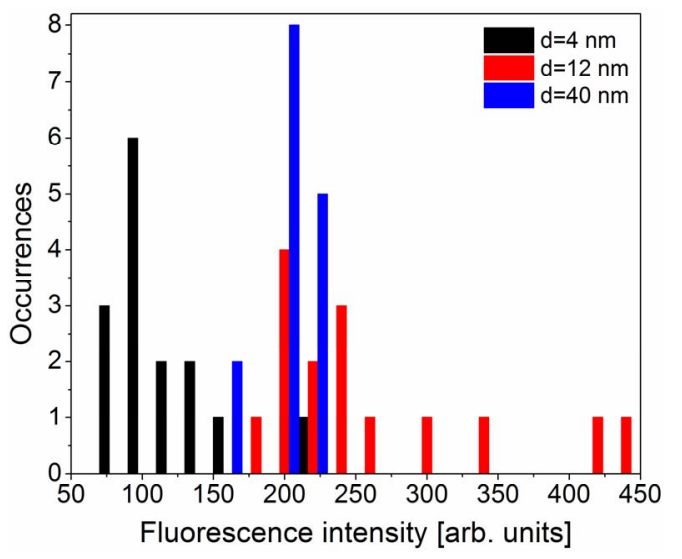

Figure 6. Histograms of the fluorescence intensity extracted by integrating the fluorescence transients. The data for hybrid nanostructures with spacer thickness of $\mathbf{4} \mathbf{~ n m}$ (black), $12 \mathrm{~nm}$ (red) and $40 \mathrm{~nm}$ (blue) are shown.

two samples indicate that for the PCP complexes deposited on Au nanoparticles, the photobleaching is substantially enhanced for the structure where the coupling is the strongest. This observation points at pretty complex nature of interactions present in plasmonic structures where many factors contribute to the final result and many processes should be taken into account.

\section{Conclusion}

We studied fluorescence properties of a hybrid nanostructure composed of spherical $\mathrm{Au}$ nanoparticles and peridinin-chlorophyll-protein light-harvesting complexes separated with a dielectric layer of $4 \mathrm{~nm}, 12 \mathrm{~nm}$, and 40 $\mathrm{nm}$ thickness. We find that the maximum enhancement of approximately 7-fold can be obtained for the 12-nmthick silica spacer. The increase of the emission intensity is accompanied with shortening of the fluorescence lifetime. The analysis of the experimental data points towards strong influence of plasmon excitations in metallic nanoparticles upon the photostability of the PCP complexes. The effect strongly depends on the spectral overlap between plasmon resonance and fluorescence of the investigated structure.

\section{Acknowledgements}

Research in Poland has been supported by the WELCOME project "Hybrid Nanostructures as a Stepping Stone towards Efficient Artificial Photosynthesis" funded by the Foundation for Polish Science and EUROCORES project "BOLDCATS" funded by the European Science Foundation.

\section{REFERENCES}

[1] J. Lakowicz, "Plasmonics in Biology and Plasmon-Con- trolled Fluorescence," Plasmonics, Vol. 1, No. 1, 2006 , pp. 5-33. doi:10.1007/s11468-005-9002-3

[2] J. Malicka, I. Gryczynski, C. D. Geddes and J. R. Lakowicz, "Metal-Enhanced Emission from Indocyaninegreen: A Newapproach to in Vivo Imaging," Journal of Biomedical Optics, Vol. 8, No. 3, 2003, pp. 472-478. doi:10.1117/1.1578643

[3] A. Hartschuh, H. N. Pedrosa, L. Novotny and T. D. Krauss, "Simultaneous Fluorescence and Raman Scattering from Single Carbon Nanotubes," Science, Vol. 301, No. 5638, 2003, pp. 1354-1356. doi:10.1126/science. 1087118

[4] J. Lee, P. Hernandez, J. Lee, A. Govorov and N. Kotov, "Exciton-Plasmon Interactions in Molecular Spring Assemblies of Nanowires and Wavelength-Based Protein Detection," Nature Materials, Vol. 6, No. 4, 2007, pp. 291-295. doi:10.1038/nmat1869

[5] A. Polman and H. A. Atwater, "Photonic Design Principles for Ultrahigh-Efficiency Photovoltaics," Nature Materials, Vol. 11, No. 3, 2012, pp. 174-177. doi:10.1038/nmat3263

[6] E. Prodan, P. Nordlander and N. J. Halas, "Electronic Structure and Optical Properties of Gold Nanoshells," Nano Letters, Vol. 3, No. 10, 2003, pp. 1411-1415. doi: $10.1021 / \mathrm{n} 1034594 \mathrm{q}$

[7] S. Eustis and M. El-Sayed, "Aspect Ratio Dependence of the Enhanced Fluorescence Intensity of Gold Nanorods: Experimental and Simulationstudy," The Journal of Physical Chemistry B, Vol. 109, No. 34, 2005, pp. 1635016356. doi:10.1021/jp052951a

[8] P. Anger, P. Bharadwaj and L. Novotny, "Enhancement and Quenching of Single-Molecule Fluorescence," Physical Review Letters, Vol. 96, No. 11, 2006, Article ID: 113002. doi:10.1103/PhysRevLett.96.113002

[9] L. Bujak, N. Czechowski, D. Piatkowski, R. Litvin, S. Mackowski, T. H. P. Brotosudarmo, R. J. Cogdell, S. Pichler and W. Heiss, "Fluorescenceenhancement of Light-Harvesting Complex 2 from Purple Bacteria Coupled to Spherical Gold Nanoparticles," Applied Physics Letters, Vol. 99, No. 17, 2011, Article ID: 173701. doi:10.1063/1.3648113

[10] A. O. Govorov, G. W. Bryant, W. Zhang, T. Skeini, J. Lee, N. A. Kotov, J. M. Slocik and R. R. Naik, "Exciton-PlasmonInteraction and Hybrid Excitons in Semiconductor-Metal Nanoparticle Assemblies," Nano Letters, Vol. 6, No. 5, 2006, pp. 984-994. doi: $10.1021 / \mathrm{nl} 0602140$

[11] M. Olejnik, L. Bujak and S. Mackowski, "Plasmonic Molecular Nanohybrids-Spectral Dependence of Fluorescence Quenching," International Journal of Molecular Sciences, Vol. 13, No. 1, 2012, pp. 1018-1028. doi:10.3390/ijms13011018

[12] K. Aslan, M. Wu, J. R. Lakowicz and C. D. Geddes, "Fluorescent Core-Shell $\mathrm{Ag} @ \mathrm{SiO}_{2}$ Nanocomposites for Metal-Enhanced Fluorescence and Single Nanoparticle Sensing Platforms," Journal of the American Chemical Society, Vol. 129, No. 6, 2007, pp. 1524-1525. doi: $10.1021 / \mathrm{ja} 0680820$

[13] K. Ray, R. Badugu and J. R. Lakowicz, "Metal-Enhanced 
Fluorescence from CdTe Nanocrystals: A Single-Molecule Fluorescence Study," Journal of the American Chemical Society, Vol. 128, No. 28, 2006, pp. 8998-8999. doi:10.1021/ja061762i

[14] S. Mackowski, S. Wormke, A. J. Maier, T. H. P. Brotosudarmo, H. Harutyunyan, A. Hartschuh, A. O. Govorov, H. Scheer and C. Brauchle, "Metal-Enhanced Fluorescence of Chlorophylls in Single Light-Harvesting Complexes," Nano Letters, Vol. 8, No. 2, 2007, pp. 558-564. doi:10.1021/n1072854o

[15] D. J. Miller, J. Catmull, R. Puskeiler, H. Tweedale, F. P. Sharples and R. G. Hiller, "Reconstitution of the Peridinin-Chlorophyll a Protein (PCP): Evidence for Functional Flexibility in Chlorophyll Binding," Photosynthesis Research, Vol. 86, No. 1, 2005, pp. 229-240. doi:10.1007/s11120-005-2067-1

[16] J. Roither, "Optical Applications of Colloidal Nanocrystals," PhD Thesis, Johannes Kepler University, Linz, 2009.

[17] B. Krajnik, T. Schulte, D. Piątkowski, N. Czechowski, E. Hofmann and S. Mackowski, "SIL-Based Confocal Fluorescence Microscope for Investigating Individual Nanos- tructures," Central European Journal of Physics, Vol. 9, No. 2, 2011, pp. 293-299.

doi:10.2478/s11534-010-0098-5

[18] T. Schulte, S. Johanning and E. Hofmann, "Structure and Function of Native and Refolded Peridinin-ChlorophyllProteins from Dinoflagellates," European Journal of Cell Biology, Vol. 89, No. 12, 2010, pp. 990-997. doi:10.1016/j.ejcb.2010.08.004

[19] F. J. Kleima, E. Hofmann, B. Gobets, I. H. M. van Stokkum, R. van Grondelle, K. Diederichs and H. van Amerongen, "Förster Excitation Energy Transfer in Peridinin-Chlorophyll-a-Protein," Biophysical Journal, Vol. 78, No. 1, 2000, pp. 344-353. doi:10.1016/S0006-3495(00)76597-0

[20] S. Wörmke, S. Mackowski, T. H. P. Brotosudarmo, C. Jung, A. Zumbusch, M. Ehrl, H. Scheer, E. Hofmann, R. G. Hiller and C. Bräuchle, "Monitoring Fluorescence of Individual Chromophores in Peridinin-Chlorophyll-Protein Complex Using Single Molecules Pectroscopy," Biochimica et Biophysica Acta, Vol. 1767, No. 7, 2007, pp. 956-964. doi:10.1016/j.bbabio.2007.05.004 